\title{
Resonant Light Absorption by Semiconductor Quantum Dots
}

\author{
I. G. Lang ${ }^{1}$ and S. T. Pavlov ${ }^{2}$ \\ ${ }^{1}$ A.F. Ioffe Physical-Technical Institute, Russian Academy of Sciences, 194021 St. Petersburg, Russia \\ ${ }^{2}$ P.N. Lebedev Physical Institute, Russian Academy of Sciences, 119991 Moscow, Russia
}

Correspondence should be addressed to S. T. Pavlov, pstanislav12@gmail.com

Received 9 January 2009; Revised 29 April 2009; Accepted 27 May 2009

Recommended by Sasha Alexandrov

The cross-section of light absorption by size-quantized semiconductor quantum dots (QD) is calculated in the case of a resonance with an exciton $\Gamma_{6} \times \Gamma_{7}$ in cubical crystals of $T_{d}$ class. The interference of stimulating and induced electric and magnetic fields is taken into account. The cross-section of light absorption is proportional to the exciton nonradiative damping $\gamma$.

Copyright ( 2009 I. G. Lang and S. T. Pavlov. This is an open access article distributed under the Creative Commons Attribution License, which permits unrestricted use, distribution, and reproduction in any medium, provided the original work is properly cited.

\section{Introduction}

Study of the resonant reflection and absorption of light by low-dimensional semiconductor objects is a simple and reliable way of the determination of exciton parameters [1-5]. When the size-quantized semiconductor objects (quantum wells (QW), quantum wires, and quantum dots (QD)) are irradiated by light, elastic light scattering and absorption intensify resonantly if the light frequency $\omega_{l}$ equals to the exciton frequency $\tilde{\omega}_{0}$. The resonant peak width is determined by the exciton damping $\Gamma=\gamma_{r}+\gamma$, which consists of nonradiative $\gamma$ and radiative $\gamma_{r}$ damping. An important role of the radiative damping $\gamma_{r}$ was proved for the first time in [6-8]. Light reflection by some structures, consisting of quantum wells, wires, and dots was considered in [9].

The light elastic scattering on a QD of arbitrary form and sizes in a resonance with excitons is investigated in [10], where the quantum perturbation theory is used. However, the quantum method does not allow to get out of the lowest order on the light-electron interaction and to calculate corrections to the exciton energy due to that interaction. Such approximation is acceptable only under condition $\gamma_{r} \ll \gamma$.

In the present work, a semiclassical method [9, 11-14] is applied for calculation of electric and magnetic fields, while a description of electrons remains quantum one. The semiclassical method allows to calculate precisely electric and magnetic fields on large distances from a QD, that is, to take into account all the orders on the light-electron interaction. It allows to introduce into the theory the nonradiative damping $\gamma$, to calculate the light absorption and the exciton energy corrections due to the long-range exchange electronhole interaction. At last, the method admits consideration of the monochromatic and pulse irradiation (see, e.g., [15]).

There are two variants of the semiclassical method. The first of them assumes using of boundary conditions for electric and magnetic fields on the semiconductor object boundaries (see, e.g., $[11,16]$ ). However, using of boundary conditions in the case of spherical QDs, for instance, realizes into cumbersome calculations [11], and calculations become much more complicate for other forms. Therefore, we use here the second variant of the semiclassical method-the method of retarded potentials_-allowing to avoid using of boundary conditions at all.

\section{The Method of Retarded Potentials}

First of all, we calculate the current $\mathbf{j}(\mathbf{r}, t)$ and charge $\rho(\mathbf{r}, t)$ densities induced by the electric field inside of the object $[12,17,18]$ and averaged on the ground state of the crystal. Using of stimulating electric field $\mathbf{E}_{0}(\mathbf{r}, t)$ in these expressions leads again to the limitation by the lowest order on the lightelectron interaction. But substitution of the genuine fields $\mathbf{E}(\mathbf{r}, t)$ inside of the object leads to the precise results.

The induced electric and magnetic fields are represented with the help of the vector $\mathbf{A}(\mathbf{r}, t)$ and scalar $\varphi(\mathbf{r}, t)$ potentials

$$
\begin{gathered}
\Delta \mathbf{E}(\mathbf{r}, t)=-\frac{1}{c} \frac{\partial \mathbf{A}(\mathbf{r}, t)}{\partial t}-\frac{\partial \varphi(\mathbf{r}, t)}{\partial \mathbf{r}}, \\
\Delta \mathbf{H}(\mathbf{r}, t)=\operatorname{rot} \mathbf{A}(\mathbf{r}, t) .
\end{gathered}
$$


The retarded potentials are [19, page 447]

$$
\begin{aligned}
& \mathbf{A}(\mathbf{r}, t)=\frac{1}{c} \int d^{3} r^{\prime} \frac{\mathbf{j}\left(\mathbf{r}^{\prime}, t-v\left|\mathbf{r}-\mathbf{r}^{\prime}\right| / c\right)}{\left|\mathbf{r}-\mathbf{r}^{\prime}\right|}, \\
& \varphi(\mathbf{r}, t)=\frac{1}{\nu^{2}} \int d^{3} r^{\prime} \frac{\rho\left(\mathbf{r}^{\prime}, t-\nu\left|\mathbf{r}-\mathbf{r}^{\prime}\right| / c\right)}{\left|\mathbf{r}-\mathbf{r}^{\prime}\right|},
\end{aligned}
$$

where $v$ is the light refraction coefficient (we assume it is identical inside and outside of a QD). Having used the current and charge densities with the precise field $\mathbf{E}(\mathbf{r}, t)=$ $\mathbf{E}_{0}(\mathbf{r}, t)+\Delta \mathbf{E}(\mathbf{r}, t)$ and substituting (2) into (1), we obtain an integral equation for the induced field $\Delta \mathbf{E}(\mathbf{r}, t)$ inside of the object. In some cases this equation may be solved. Having calculated the precise field inside of the object, we obtain the fields outside with the help of (2) and (1).

\section{The Induced Current Density}

The quantum theory of conductivity of low-dimensional objects is elaborated in $[17,18,20]$. The average current and charge densities induced by a weak spatially inhomogeneous electromagnetic field are calculated for the spatially inhomogeneous systems. It was shown that the averaged current and charge densities contain two contributions, first of which is expressed through the electric field, and the second is expressed through the first spatial derivatives of the electric field. Situations when the second contribution may be neglected are discussed in [20]. The main contribution into the average current density at temperature $T=0$ is as follows:

$$
\begin{aligned}
j_{\alpha}(\mathbf{r}, t)= & \frac{i}{\hbar} \int d^{3} r^{\prime} \int_{-\infty}^{t} d t^{\prime} \\
& \times\left\langle 0\left|\left[\hat{j}_{\alpha}(\mathbf{r}, t), \bar{d}_{\beta}\left(\mathbf{r}^{\prime}, \mathbf{t}^{\prime}\right)\right]\right| 0\right\rangle E_{\beta}\left(\mathbf{r}^{\prime}, \mathbf{t}^{\prime}\right)+\text { c.c. }
\end{aligned}
$$

where determinations of [11] are used: $\langle 0|\cdots| 0\rangle$ is the average value on the ground state, $[\hat{a}, \hat{b}]$ is the commutator of operators $\hat{a}$ and $\hat{b}, \hat{\mathbf{j}}(\mathbf{r}, t)$ is the operator of current density in the interaction representation, $\overline{\mathbf{d}}(\mathbf{r})=\sum_{i} \overline{\mathbf{r}}_{i} \rho_{i}(\mathbf{r}), \overline{\mathbf{r}}_{i}=$ $\mathbf{r}_{i}-\left\langle 0\left|\mathbf{r}_{i}\right| 0\right\rangle, \rho_{i}(\mathbf{r})=e \delta\left(\mathbf{r}-\mathbf{r}_{i}\right), \mathbf{E}(\mathbf{r}, t)$ is the classic electric field.

We assume $T=0$. It was assumed also in (3) that currents and charges are absent at infinite distances, and that the electromagnetic field equals 0 at $t \rightarrow-\infty$, what corresponds to adiabatic switching on of fields. We suppose an interaction between charged particles, a possible presence of an external potential and quantizing magnetic field.

We apply (3) to consideration of low-dimensional sizequantized semiconductor objects [7] and suppose that the stimulating light frequency or carrying frequency of a pulse irradiation are close to the semiconductor energy gap $\hbar \omega_{g}$. Sizes $R$ of a semiconductor object are much more than a lattice constant $R \gg a$. The distances, on which the smooth multiplier from the wave function varies, are much greater $a$ and comparable to the sizes of the object. Then the effective mass approximation is applicable and the sizequantization condition is satisfied. The last statement means that the exciton energy spectrum is discrete one. Then $[7,8]$, the average density of induced current in QWs, QDs, and quantum wires is determined by the expression

$$
\begin{aligned}
\mathbf{j}(\mathbf{r}, t)= & \frac{i e^{2}}{2 \pi \hbar \omega_{g} m_{0}^{2}} \sum_{\eta} \\
& \times\left\{\mathbf{p}_{c v \eta}^{*} F_{\eta}(\mathbf{r}) \int_{-\infty}^{+\infty} d \omega \frac{e^{-i \omega t}}{\omega-\omega_{\eta}+i \gamma_{\eta} / 2}\right. \\
& \times \int d \mathbf{r}^{\prime} F_{\eta}^{*}\left(\mathbf{r}^{\prime}\right)\left(\mathbf{p}_{c v \eta} \mathcal{E}\left(\mathbf{r}^{\prime}, \omega\right)\right) \\
& +\mathbf{p}_{c v \eta} F_{\eta}^{*}(\mathbf{r}) \int_{-\infty}^{+\infty} d \omega \frac{e^{-i \omega t}}{\omega+\omega_{\eta}+i \gamma_{\eta} / 2} \\
& \left.\times \int d \mathbf{r}^{\prime} F_{\eta}\left(\mathbf{r}^{\prime}\right)\left(\mathbf{p}_{c v \eta}^{*} \mathcal{E}\left(\mathbf{r}^{\prime}, \omega\right)\right)\right\}+c . c .,
\end{aligned}
$$

where the determinations are used: $e=-|e|, m_{0}$ are the electron charge and mass, respectively, $p_{c v \eta}$ is the interband matrix element of quasimomentum operator, corresponding to the exciton with indexes $\eta, F_{\eta}(\mathbf{r})$ is the envelope exciton wave function at $\mathbf{r}_{e}=\mathbf{r}_{h}=\mathbf{r}$, where $\mathbf{r}_{e}\left(\mathbf{r}_{h}\right)$ is the electron (hole) radius-vector, $\hbar \omega_{\eta}$ is the $\eta$ exciton energy counted from the ground state energy, $\gamma_{\eta}$ is the nonradiative exciton damping. The set $\eta$ includes indexes of semiconductor valence and conduction bands, and 6 indexes, characterizing an exciton in the effective mass approximation (these 6 indexes describe the function $\left.F_{\eta}(\mathbf{r})\right)$. Finally, $\mathcal{E}(\mathbf{r}, \omega)$ ) is the Fourier transform of the electric field

$$
\mathbf{E}(r, t)=\frac{1}{2 \pi} \int_{-\infty}^{\infty} d \omega e^{-i \omega t} \mathcal{E}(\mathbf{r}, \omega)+c . c .
$$

The main and conjugated contributions in (5) are chosen so that

$$
\mathcal{E}_{0}(\mathbf{r}, \omega)=2 \pi E_{0} \mathbf{e}_{l} e^{i \mathbf{k r}} D_{0}(\omega),
$$

where $\mathbf{k}$ is the wave vector of stimulating light, the function $D_{0}(\omega)$ describes the form of a stimulating pulse [12]. The average density of charge induced inside of a semiconductor object may be determined with the help of (4) and continuity equation

$$
\operatorname{div} \mathbf{j}(\mathbf{r}, t)+\frac{\partial \rho(\mathbf{r}, t)}{\partial t}=0
$$

\section{The Case of the Exciton $\Gamma_{6} \times \Gamma_{7}$ in a QD}

Let us consider an exciton consisting of an electron from twofold degenerated conductive band $\Gamma_{6}$ and a hole from twofold degenerated valence band $\Gamma_{7}$ chipped of by the spinorbital interaction in $T_{d}$ class crystals. The exciton $\Gamma_{6} \times$ $\Gamma_{7}$ (see $\left.[10,11]\right)$ is the most simple object in contrast to excitons containing light and heavy holes. All the measurable values do not depend on the direction of vectors relatively of crystallographic axes, that is, a crystal plays a role of an isotropic medium.

According to determinations of [21, page 73], the electron wave functions have the structure

$$
\Psi_{c 1}=i S \uparrow, \quad \Psi_{c 2}=i S \downarrow,
$$


and the hole wave functions are

$$
\begin{aligned}
& \Psi_{h 1}=\frac{1}{\sqrt{3}}(X-i Y) \uparrow-\frac{1}{\sqrt{3}} Z \downarrow, \\
& \Psi_{h 2}=\frac{1}{\sqrt{3}}(X+i Y) \downarrow+\frac{1}{\sqrt{3}} Z \uparrow .
\end{aligned}
$$

Having combined (8) and (9) in pairs, we obtain fourfould degenerated excitonic state for which the interband matrix elements of momentum operator result in

$$
\begin{aligned}
\mathbf{p}_{c v 1}=\frac{p_{c v}}{\sqrt{3}}\left(\mathbf{e}_{x}-i \mathbf{e}_{y}\right), & \mathbf{p}_{c v 2}=\frac{p_{c v}}{\sqrt{3}}\left(\mathbf{e}_{x}+i \mathbf{e}_{y}\right), \\
\mathbf{p}_{c v 3}=\frac{p_{c v}}{\sqrt{3}} \mathbf{e}_{z}, & \mathbf{p}_{c v 4}=-\frac{p_{c v}}{\sqrt{3}} \mathbf{e}_{z},
\end{aligned}
$$

where the scalar

$$
p_{c v}=i\left\langle S\left|\hat{p}_{x}\right| X\right\rangle
$$

is introduced; $\mathbf{e}_{x}, \mathbf{e}_{y}, \mathbf{e}_{z}$ are the unite vectors along the crystallographic axes.

We assume that the QD radius $R$ is much less than the bulk exciton Bohr radius (strong-confinement regime). Under these conditions the binding energy of the exciton, determined by the Coulomb interaction, may be neglected. From the four exciton states, to which the interband matrix elements (10) of the quasimomentum correspond, it is easy (by drawing up the linear combinations) to obtain three bright and one dark excitonic states, about which the speech goes, for example, in $[11,22,23]$. Below we show that the corrections to the bright exciton energy, caused by longrange exchange interaction of electrons and holes, occur in our theory automatically and coincide with the results of $[11,22,23]$.

\section{Electric and Magnetic Fields on Large Distances from a QD}

Let us consider light scattering by a QD near the resonance with some energy level of $\Gamma_{6} \times \Gamma_{7}$ exciton, when other excitons and excitonic energy levels may be neglected. Our energy level is nondegenerated, then the index $\eta$ takes on four values from 1 to 4 , and only the values $\mathbf{p}_{c v \eta}$, according to (10), depend on $\eta$. Without light-electron interaction we have

$$
\begin{gathered}
\omega_{\eta}=\omega_{0}, \\
\gamma_{\eta}=\gamma, \quad F_{\eta}(\mathbf{r})=F(\mathbf{r}),
\end{gathered}
$$

though the function $F(\mathbf{r})$ may be chosen as a real one. After summation on $\eta$ in (4) with using (10), we obtain

$$
\mathbf{j}(\mathbf{r}, t)=\frac{i e^{2} p_{c v}^{2}}{3 \pi \hbar \omega_{g} m_{0}^{2}} F(\mathbf{r}) \int_{-\infty}^{\infty} d \omega e^{-i \omega t} \mathbf{T}(\omega) L(\omega)+\text { c.c. },
$$

where

$$
\begin{aligned}
& \mathbf{T}(\omega)=\int d^{3} r \mathcal{E}(\mathbf{r}, \omega) F(\mathbf{r}), \\
& L(\omega)=\frac{1}{\omega-\omega_{0}+i \gamma / 2}+\frac{1}{\omega+\omega_{0}+i \gamma / 2} .
\end{aligned}
$$

With the help of (7) we obtain the induced charge density

$$
\rho(\mathbf{r}, t)=\frac{e^{2} p_{c v}^{2}}{3 \pi \hbar \omega_{g} m_{0}^{2}} \int_{-\infty}^{\infty} \frac{d \omega}{\omega} e^{-i \omega t}\left(\frac{d F(\mathbf{r})}{d \mathbf{r}}\right) \mathbf{T}(\omega) L(\omega)+c . c .
$$

The nonresonant term from (14) is neglected below.

Using (2), we obtain electric and magnetic fields on large distances from a QD

$$
\begin{aligned}
\left.\Delta \mathbf{E}_{c}(\mathbf{r}, t)\right|_{r \rightarrow \infty} & =E^{+}(\mathbf{r}, t) \mathbf{e}_{s}^{+}+E^{-}(\mathbf{r}, t) \mathbf{e}_{s}^{-}+c . c ., \\
\left.\Delta \mathbf{H}_{c}(\mathbf{r}, t)\right|_{r \rightarrow \infty} & =H^{+}(\mathbf{r}, t) \mathbf{e}_{s}^{+}+H^{-}(\mathbf{r}, t) \mathbf{e}_{s}^{-}+c . c .,
\end{aligned}
$$

where

$$
\begin{gathered}
E^{ \pm}(\mathbf{r}, t)=-\frac{e^{2}}{2 \pi \hbar \omega_{g} m_{0}^{2} c^{2} r} \int_{-\infty}^{\infty} d \omega \omega e^{i(k r-\omega t)} Q\left(\mathbf{k}_{s}, \omega, \mathbf{e}_{s}^{ \pm}\right), \\
H^{ \pm}(\mathbf{r}, t)=\mp i v E^{ \pm}(\mathbf{r}, t), \\
Q\left(\mathbf{k}_{s}, \omega, \mathbf{e}_{s}^{ \pm}\right)=\frac{2}{3} p_{c v}^{2} P\left(\mathbf{k}_{s}\right)\left(\mathbf{T}(\omega) \mathbf{e}_{s}^{\mp}\right) L(\omega), \\
P(\mathbf{k})=\int d^{3} r e^{-i \mathbf{k r}} F(\mathbf{r}),
\end{gathered}
$$

$\mathbf{k}_{s}=(\omega \nu / c)(\mathbf{r} / r)$ is the wave vector of scattered light.

To obtain precise induced electric and magnetic fields on large distances from a $\mathrm{QD}$, we have to calculate the vector $\mathbf{T}(\omega)$, determined by the genuine electric field inside of the QD and consisting of stimulating and induced fields

$$
\mathbf{E}(\mathbf{r}, t)=E_{0} \mathbf{e}_{l} \int_{-\infty}^{\infty} d \omega e^{i(\mathbf{k r}-\omega t)} D_{0}(\omega)+\Delta \mathbf{E}(\mathbf{r}, t),
$$

where $k=\omega \nu / c, \mathbf{e}_{l}$ is the circular polarization vector. In the case of a monochromatic irradiation

$$
D_{0}(\omega)=\delta\left(\omega-\omega_{l}\right),
$$

but at a pulse irradiation the frequency $\omega$ is widespread in the interval $\Delta \omega$ near $\omega_{l}$, and $\Delta \omega$ is vice inverse to the pulse duration $\Delta t$.

Using the formulas for retarded potentials, we obtain the induced field

$$
\begin{aligned}
\mathcal{E}(\mathbf{r}, \omega)= & 2 \pi \mathbf{e}_{l} E_{0} e^{i \mathbf{k r}} D_{0}(\omega)-\frac{2 e^{2}}{3 \hbar c} \frac{p_{c v}^{2} \omega}{\omega_{g} m_{0}^{2} c} \\
& \times L(\omega)\left[\mathbf{T}(\omega)+\frac{1}{k^{2}}\left(\mathbf{T}(\omega) \frac{d}{d \mathbf{r}}\right) \frac{d}{d \mathbf{r}}\right] \Phi(\mathbf{r}),
\end{aligned}
$$

where

$$
\Phi(\mathbf{r})=\int d^{3} r^{\prime} F_{\eta}\left(\mathbf{r}^{\prime}\right) \frac{e^{i k\left|\mathbf{r}-\mathbf{r}^{\prime}\right|}}{\left|\mathbf{r}-\mathbf{r}^{\prime}\right|} .
$$

Equation (20) is an integral equation for $\mathcal{E}(\mathbf{r}, \omega)$. Then

$$
\begin{gathered}
\Phi(\mathbf{r})=\frac{1}{(2 \pi)^{3}} \int d^{3} q e^{i \mathbf{q r}} P(\mathbf{q})\left\{\frac{4 \pi P}{q^{2}-k^{2}}+\frac{2 i \pi^{2}}{k} \delta(q-k)\right\}, \\
\frac{P}{a-b}=\frac{1}{2}\left(\frac{1}{a-b+i \delta}+\frac{1}{a-b-i \delta}\right), \quad \delta \longrightarrow+0 .
\end{gathered}
$$


Let us substitute (22) in (20), multiply both sides on $F(\mathbf{r})$, and integrate on $\mathbf{r}$. We obtain the equation for the vector $\mathbf{T}(\omega)$ :

$$
\begin{aligned}
\mathbf{T}(\omega)(1 & \left.+C(\omega) \int d^{3} q J(\mathbf{q})\right) \\
= & 2 \pi E_{0} \mathbf{e}_{l} D_{0}(\omega) P^{*}(\mathbf{k}) \\
& +\frac{C(\omega)}{k^{2}} \int d^{3} q \mathbf{q}(\mathbf{q} \mathbf{T}(\omega)) J(\mathbf{q})
\end{aligned}
$$

where determinations

$$
\begin{gathered}
C(\omega)=\frac{2}{3}\left(\frac{e^{2}}{\hbar c}\right) \frac{p_{c v}^{2} \omega}{\omega_{g} m_{0}^{2} c} L(\omega), \\
J(\mathbf{q})=|P(\mathbf{q})|^{2}\left[\frac{1}{2 \pi^{2}} \frac{P}{q^{2}-k^{2}}+\frac{i}{4 \pi k} \delta(q-k)\right],
\end{gathered}
$$

are introduced.

Equation (23) may be considered as a system of three equations for the components $T_{x}(\omega), T_{y}(\omega), T_{z}(\omega)$. Having solved this system, we can substitute the results into expressions for induced fields on large distances from the QD. Thus, the problem of light scattering in the resonance with an exciton $\Gamma_{6} \times \Gamma_{7}$ in a QD of arbitrary form is solved in principal at any relation of the light wave length and $Q D$ 's sizes.

\section{The Special Case of a Spherical Symmetrical Envelope Wave Function}

Let us consider a special case when the system of three equations is reduced to one equation. It happens if the function $P(\mathbf{k})$ depends only on the module $k$, that is,

$$
P(\mathbf{k})=P(k) .
$$

The condition (25) is fulfilled, if the function $F(\mathbf{r})$ is spherically symmetrical or the QD sizes are much less than the wave length of the stimulating light when $P(\mathbf{k})=P(0)$. For instance, (25) is satisfied in the case of a spherical QD limited by the infinitely high rectangular potential barrier. Then the envelope wave function

$$
F(r)=\frac{1}{2 \pi R} \frac{\sin ^{2}(\pi r / R)}{r^{2}} \theta(R-r)
$$

corresponds to the lowest excitonic energy level $\omega_{0}$, and

$$
P(k)=\frac{2}{k R} \int_{0}^{\pi} d x \sin \frac{k R x}{\pi} \frac{\sin ^{2} x}{x}, \quad P(0)=1 .
$$

Then

$$
\mathbf{T}(\omega)=\frac{2 \pi E_{0} \mathbf{e}_{l} D_{0}(\omega) P^{*}(k)\left(\omega-\omega_{0}+i \gamma / 2\right)}{\omega-\widetilde{\omega}_{0}+i\left(\gamma+\gamma_{r}\right) / 2}
$$

where

$$
\begin{aligned}
\gamma_{r}= & \frac{8 v}{9} \frac{e^{2}}{c \hbar} \frac{p_{c v}^{2} \omega^{2}}{\omega_{g} m_{0}^{2} c^{2}}|P(k)|^{2}, \\
\tilde{\omega}_{0}= & \omega_{0}+\Delta \omega \\
\Delta \omega= & -\frac{4 e^{2}}{9 \pi \hbar} \frac{p_{c v}^{2}}{\omega_{g} \omega m_{0}^{2} v^{2}} \int_{0}^{\infty} d q q^{2}(P(q))^{2} \\
& \times\left(q^{2}-3 k^{2}\right) \frac{P}{q^{2}-k^{2}} .
\end{aligned}
$$

The value (30) of an exciton energy correction coincides completely with the results of [6] and preceding results of [16-18], where it was considered as the result of the longrange exchange electron-hole interaction (see [8]). Having substituted (28) in (16) and (17), we obtain electric and magnetic fields on the large distances from the QD applicable in the cases of the monochromatic or pulse irradiation.

The precise results for the fields distinguish from the results of the lowest approximation on the light-electron interaction only by the substitution $\omega_{0}$ by $\widetilde{\omega}_{0}$ and $\gamma$ by $\Gamma=$ $\gamma+\gamma_{r}$.

For the case (19) of the monochromatic irradiation we obtain

$$
\begin{aligned}
\left.\Delta \mathbf{E}((\mathbf{r}), t)\right|_{r \rightarrow \infty}= & -\frac{3}{4} E_{0} \frac{\gamma_{r}}{k_{l} r}\left[\left(\mathbf{e}_{l} \mathbf{e}_{s}^{+}\right) \mathbf{e}_{s}^{+}+\left(\mathbf{e}_{l} \mathbf{e}_{s}^{+}\right) \mathbf{e}_{s}^{-}\right] \\
& \times \frac{e^{i\left(k_{l} r-\omega_{l} t\right)}}{\omega_{l}-\widetilde{\omega}_{0}+i \Gamma / 2}+c . c ., \\
\left.\Delta \mathbf{H}((\mathbf{r}), t)\right|_{r \rightarrow \infty}= & \frac{3 i v}{4} E_{0} \frac{\gamma_{r}}{k_{l} r}\left[\left(\mathbf{e}_{l} \mathbf{e}_{s}^{-}\right) \mathbf{e}_{s}^{+}-\left(\mathbf{e}_{l} \mathbf{e}_{s}^{+}\right) \mathbf{e}_{s}^{-}\right] \\
& \times \frac{e^{i\left(k_{l} r-\omega_{l} t\right)}}{\omega_{l}-\widetilde{\omega}_{0}+i \Gamma / 2}+\text { c.c. },
\end{aligned}
$$

where $k_{l}=\omega_{l} \nu / c$.

\section{The Pointing Vector on Large Distances from the QD and the Total Cross-Section of Light Scattering at the Monochromatic Irradiation}

The Pointing vector on large distances from the QD equals

$$
\left.\mathbf{S}\right|_{r \rightarrow \infty}=\mathbf{S}_{0}+\mathbf{S}_{\text {inter } f}+\mathbf{S}_{\text {scat }}
$$

where

$$
\begin{aligned}
\mathbf{S}_{0} & =\frac{c}{4 \pi} \mathbf{E}_{0} \times \mathbf{H}_{0}=\frac{c \nu}{2 \pi} E_{0}^{2} \mathbf{e}_{z}, \\
\mathbf{S}_{\text {inter } f} & =\frac{c}{4 \pi}\left[\mathbf{E}_{0} \times \Delta \mathbf{H}+\Delta \mathbf{E} \times \mathbf{H}_{0}\right], \\
\mathbf{S}_{\text {scat }} & =\frac{c}{4 \pi} \Delta \mathbf{E} \times \Delta \mathbf{H} .
\end{aligned}
$$

With the help of (31) we obtain

$$
\mathbf{S}_{\mathrm{scat}}=\frac{9 \pi}{4} S_{0} \frac{\gamma_{r}^{2}}{\left(k_{l} r\right)^{2}} \frac{\mathbf{r}}{r} \frac{\left|\mathbf{e}_{l} \mathbf{e}_{s}^{-}\right|^{2}+\left|\mathbf{e}_{l} \mathbf{e}_{s}^{+}\right|^{2}}{\left(\omega_{l}-\tilde{\omega}_{0}\right)^{2}+\Gamma^{2} / 4} .
$$


The module of the total flux of scattered light per time unite is

$$
\Pi_{\mathrm{scat}}=\frac{3 \pi}{2} S_{0} \frac{\gamma_{r}^{2}}{\left(k_{l}\right)^{2}} \frac{1}{\left(\omega_{l}-\tilde{\omega}_{0}\right)^{2}+\Gamma^{2} / 4} .
$$

Obtaining (35) from (34) we have used the relations

$$
\begin{aligned}
& \left|\mathbf{e}_{l}^{+} \mathbf{e}_{s}^{-}\right|^{2}=\left|\mathbf{e}_{l}^{-} \mathbf{e}_{s}^{+}\right|^{2}=\frac{1}{4}(1+\cos \theta)^{2}, \\
& \left|\mathbf{e}_{l}^{+} \mathbf{e}_{s}^{+}\right|^{2}=\left|\mathbf{e}_{l}^{-} \mathbf{e}_{s}^{-}\right|^{2}=\frac{1}{4}(1-\cos \theta)^{2},
\end{aligned}
$$

where $\theta$ is the scattering angle. An integration $\theta$ is performed. Having divided $\Pi_{\text {scat }}$ on the density $S_{0}$ of the stimulating light flux and using $k_{l}=2 \pi / \lambda_{l}$, we obtain the total cross-section of light scattering

$$
\sigma_{\text {scat }}=\frac{3}{2 \pi} \lambda_{l}^{2} \frac{\gamma_{r}^{2} / 4}{\left(\omega_{l}-\tilde{\omega}_{0}\right)^{2}+\Gamma^{2} / 4}
$$

The differential cross-sections have been determined in [8].

\section{The Light Absorption: The Role of the Nonradiative Damping}

The results for the cross-sections of light scattering on QD, obtained with the help of the quasiclassic method, coincide with the lowest approximation on the light-electron interaction with the results of the quantum perturbation theory [10]. However, the quasiclassic method allows to obtain also the cross-section of light absorption by a QD. In the case of a monochromatic irradiation the absorption is stipulated by the nonradiative damping $\gamma$ of excitons, and it equals 0 at $\gamma=0$. The same result was obtained at a monochromatic irradiation of a $\mathrm{QW}[7,24]$. The cause is that at $\gamma=0$ a dissipation of energy, spent on an exciton creation, is absent. And this energy returns at an exciton annihilation. In the case of a pulse irradiation the integral absorption equals 0 at $\gamma=0[15,25-27]$.

It was shown at calculations of light absorption by a QW that the interference of stimulating and induced electromagnetic fields must be taken into account (see, e.g., [25]). The same is true for the case of QDs.

\section{The Interference Contribution into Energy Fluxes}

Let us calculate an interference contribution into the Pointing vector at $r \rightarrow \infty$ in the case of a monochromatic irradiation. Using (31) and

$$
\begin{gathered}
\mathbf{E}_{0}^{ \pm}(\mathbf{r}, t)=E_{0} \mathbf{e}_{l}^{ \pm} e^{i\left(\mathbf{k}_{l} \mathbf{r}-\omega_{l} t\right)}+c . c ., \\
\mathbf{H}_{0}^{ \pm}(\mathbf{r}, t)=E_{0} \nu\left[\mathbf{e}_{z} \times \mathbf{e}_{l}^{ \pm}\right] e^{i\left(\mathbf{k}_{l} \mathbf{r}-\omega_{l} t\right)}+c . c .,
\end{gathered}
$$

we obtain

$$
\begin{gathered}
\mathbf{S}_{\text {inter } f}=\mathbf{S}_{z}+\mathbf{S}_{\perp} \\
\mathbf{S}_{z}=-\frac{3}{4} \frac{\gamma_{r}}{k_{l} r} S_{0} \mathbf{e}_{z}\left|\mathbf{e}_{l}^{+} \mathbf{e}_{s}^{-}\right|^{2}\left(\frac{e^{i\left(\mathbf{k}_{l} \mathbf{r}-k_{l} r\right)}}{\omega_{l}-\widetilde{\omega}_{0}-i \Gamma / 2}+c . c .\right) \\
\mathbf{S}_{\perp}=\frac{3}{4} \frac{\gamma_{r}}{k_{l} r} S_{0}\left(\mathbf{e}_{l}^{ \pm}\left(\mathbf{e}_{l}^{\mp} \mathbf{e}_{s}^{ \pm}\right)\left(\mathbf{e}_{s}^{\mp} \mathbf{e}_{z}\right) \frac{e^{i\left(\mathbf{k}_{l} \mathbf{r}-k_{l} r\right)}}{\omega_{l}-\widetilde{\omega}_{0}-i \Gamma / 2}+\text { c.c. }\right)
\end{gathered}
$$

where $+(-)$ correspond to the right (left) circular polarization of the stimulating light.

Since (40) and (41) are applicable at $r \rightarrow \infty$, it is obviously that due to the factor $e^{i\left(\mathbf{k}_{l} \mathbf{r}-k_{l} r\right)}$ only angles $\theta \rightarrow 0$ can contribute into the constant energy flux. However, there is the factor $\left(\mathbf{e}_{l}^{\mp} \mathbf{e}_{z}\right)$ in the RHS of (41), which equals 0 at $\theta=0$. Therefore, the constant energy flux on the large distances from a QD corresponds only to the vector $\mathbf{S}_{z}$.

Let us calculate the energy flux

$$
\Pi_{z}=\int d s \mathbf{S}_{z},
$$

going through a plane perpendicular to the stimulating light direction $z$ in a time unite on large distances behind of a QD. A surface element equals $d s=\rho d \rho d \varphi$, and $\rho=z \tan \theta$, $r=z / \cos \theta$, where $\theta$ is the angle between $\mathbf{k}_{l}$ and $\mathbf{r}$. Having integrated on the angle $\theta$ which gives $2 \pi$, using (36) and going from the variable $\rho$ to the variable $\theta$, we obtain

$$
\begin{aligned}
\boldsymbol{\Pi}_{z}= & -\frac{3 \pi}{8} \frac{\gamma_{r} z}{k_{l}} \mathbf{e}_{z} \frac{\mathbf{S}_{0}}{\omega_{l}-\widetilde{\omega}_{0}-i \Gamma / 2} \int_{0}^{\pi / 2} d \theta \sin \theta \\
& \times\left(\frac{1+\cos \theta}{\cos \theta}\right)^{2} e^{i k_{l} z(1-1 / \cos \theta)}+\text { c.c. }
\end{aligned}
$$

Further we substitute variable $\theta$ by the variable $t=$ $(\cos \theta)^{-1}-1$ and obtain

$$
\begin{aligned}
\Pi_{z}= & -\frac{3 \pi}{8} \frac{\gamma_{r} z}{k_{l}} \mathbf{e}_{z} \frac{S_{0}}{\omega_{l}-\widetilde{\omega}_{0}-i \Gamma / 2} \\
& \times \int_{0}^{\infty} d t\left(\frac{2+t}{1+t}\right)^{2} e^{-i k_{l} z t}+\text { c.c. }
\end{aligned}
$$

At $z \rightarrow \infty$

$$
\begin{aligned}
z \int_{0}^{\infty} d t\left(\frac{2+t}{1+t}\right)^{2} e^{-i k_{l} z t} & \\
& \longrightarrow 4 z \int_{0}^{\infty} d t e^{-i k_{l} z t}=-\frac{4 i}{k_{l}}\left(1-e^{-i k_{l} z}\right),
\end{aligned}
$$

and we obtain

$$
\begin{aligned}
\Pi_{z} & =\frac{3 \pi}{2} \frac{\gamma_{r}}{k_{l}^{2}} \mathbf{e}_{z} S_{0}\left(\frac{i}{\omega_{l}-\tilde{\omega}_{0}-i \Gamma / 2}+c . c .\right) \\
& =-\frac{3 \pi}{2} \frac{\mathbf{e}_{z}}{k_{l}^{2}} S_{0} \frac{\gamma_{r} \Gamma}{\left(\omega_{l}-\tilde{\omega}_{0}\right)^{2}+\Gamma^{2} / 4} .
\end{aligned}
$$

In (46), the terms going to 0 at $z \rightarrow \infty$, and rapidly oscillating with $z$ are neglected. Analogically we calculate the contribution $\boldsymbol{\Pi}_{\perp}=\int d s \boldsymbol{S}_{\perp}^{ \pm}$into the energy flux and find that at $z \rightarrow \infty$ the value $\Pi_{\perp} \rightarrow 0$. 


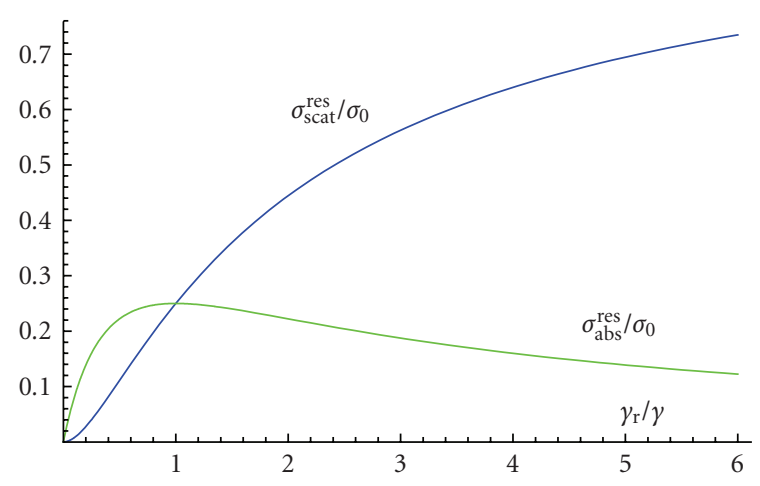

FIGURE 1: $\sigma_{\text {scat }}^{\text {res }}$ and $\sigma_{\mathrm{abs}}^{\text {res }}$ as functions of $\gamma_{r} / \gamma$ in the resonance $\widetilde{\omega}_{0}=\omega_{l}$. $\sigma_{0}=(3 / 2 \pi) \lambda_{l}^{2}$.

\section{The Cross-Section of Light Absorption}

Since $\Gamma=\gamma+\gamma_{r}$, the flux $\Pi_{z}$ may be represented as two parts

$$
\Pi_{z}=-\mathbf{e}_{z} \Pi_{\mathrm{scat}}-\mathbf{e}_{z} \Pi_{\mathrm{abs}}
$$

where $\Pi_{\text {scat }}$ is determined by (35),

$$
\Pi_{\mathrm{abs}}=\frac{3 \pi}{2} S_{0} \frac{\gamma_{r} \gamma / k_{l}^{2}}{\left(\omega_{l}-\tilde{\omega}_{0}\right)^{2}+\Gamma^{2} / 4} .
$$

Obviously that the energy flux $-\mathbf{e}_{z} \Pi_{\text {scat }}$ compensates the total flux of the scattered energy, and $\Pi_{\mathrm{abs}}$ corresponds to the energy, absorbed by the QD per time unite at the monochromatic irradiation. Having divided $\Pi_{\mathrm{abs}}$ on the density $S_{0}$ of the stimulating energy flux, we obtain the total cross-section of light absorption

$$
\sigma_{\mathrm{abs}}=\frac{3}{2 \pi} \lambda_{l}^{2} \frac{\gamma_{r} \gamma / 4}{\left(\omega_{l}-\tilde{\omega}_{0}\right)^{2}+\Gamma^{2} / 4}
$$

and reduces to 0 at $\gamma=0$, as it was supposed in advance.

Comparing (49) and (37) we find that in the lowest approximation on the light-electron interaction the absorption cross-section is of the first order on that interaction (it contains the factor $e^{2} / c \hbar$ ), and the scattering cross-section is of the second order on that interaction (it contains the factor $\left.\left(e^{2} / c \hbar\right)^{2}\right)$. The ratio of the scattering and absorption cross-sections equals $\gamma_{r} / \gamma$. The absorption cross-section has the maximum value at comparable values $\gamma_{r}$ and $\gamma$. At $\gamma_{r}=\gamma$ in the resonance $\omega_{l}=\widetilde{\omega}_{0}$

$$
\sigma_{\mathrm{scat}}^{\mathrm{res}}=\sigma_{\mathrm{abs}}^{\mathrm{res}}=\frac{3}{8 \pi} \lambda_{l}^{2} .
$$

$\sigma_{\text {scat }}^{\text {res }}$ and $\sigma_{\text {abs }}^{\text {res }}$ as functions of $\gamma_{r} / \gamma$ are represented in Figure 1.

\section{Conclusion}

Thus, the electric and magnetic fields induced at the light irradiation of QDs are calculated in the resonance of the stimulating light and excitons with the help of the semiclassical method of the retarded potentials. The fields on the large distances are calculated exactly.
The concrete calculations are performed for the excitons $\Gamma_{6} \times \Gamma_{7}$ in cubic crystals of $T_{d}$ class. It is shown that in the case of small or spherically symmetric QDs the light-electron interaction realizes into the substitution of the nonradiative excitonic damping $\gamma$ by the damping $\Gamma=\gamma+\gamma_{r}$ and the exciton energy $\omega_{0}$ by the energy $\widetilde{\omega}_{0}=\omega_{0}+\Delta \omega$, where $\gamma_{r}$ is the radiative damping, $\Delta \omega$ is the correction to the exciton energy due to the long-range exchange interaction of electrons and holes.

The light scattering and absorption cross-sections by QDs are obtained for arbitrary QDs under condition $R \ll \lambda_{l}$ and excitonic spherical envelope wave functions.

\section{References}

[1] L. Schultheis and K. Ploog, "Reflectance of two-dimensional excitons in GaAs-AlGaAs quantum wells," Physical Review B, vol. 30, no. 2, pp. 1090-1093, 1984.

[2] E. L. Ivchenko, P. S. Kop'ev, V. P. Kochereshko, et al., Fizika $i$ Technika Poluprovodnikov, vol. 22, p. 784, 1988, Physics and Technics of Semiconductors, 1988.

[3] E. L. Ivchenko, V. P. Kochereshko, P. S. Kop'ev, V. A. Kosobukin, I. N. Uraltsev, and D. R. Yakovlev, "Exciton longitudinal-transverse splitting in GaAs/AlGaAs superlattices and multiple quantum wells," Solid State Communications, vol. 70, no. 5, pp. 529-534, 1989.

[4] E. L. Ivchenko, V. P. Kochereshko, and I. N. Ural'tsev, vol. 15, Nova Science, New York, NY, USA, Studies in A.F.Ioffe Physico-Technical Institute.

[5] E. L. Ivchenko, Physics of the Solid State, vol. 33, p. 1344, 1991, translated from Fizika Tverdogo Tela, vol. 33, p. 2388, 1991.

[6] L. C. Andreani, F. Tassone, and F. Bassani, "Radiative lifetime of free excitons in quantum wells," Solid State Communications, vol. 77, no. 9, pp. 641-645, 1991.

[7] L. C. Andreani, G. Panzarini, A. V. Kavokin, and M. R. Vladimirova, "Effect of inhomogeneous broadening on optical properties of excitons in quantum wells," Physical Review B, vol. 57, no. 8, pp. 4670-4680, 1998.

[8] E. Burstein and C. Weisbuch, Eds., Confined Electrons and Photons, Plenum Press, New York, NY, USA, 1995.

[9] E. L. Ivchenko and A. V. Kavokin, Physics of the Solid State, vol. 34, p. 1815, 1992.

[10] I. G. Lang, L. I. Korovin, and S. T. Pavlov, "Elastic light scattering by semiconductor quantum dots of arbitrary shape," Physics of the Solid State, vol. 49, no. 7, pp. 1368-1374, 2007, translated from Fizika Tverdogo Tela, vol. 49, no. 7, pp. 13041309, 2007.

[11] S. V. Goupalov and E. L. Ivchenko, "Electron-hole longrange exchange interaction in semiconductor quantum dots," Journal of Crystal Growth, vol. 184-185, pp. 393-397, 1998.

[12] I. G. Lang, S. T. Pavlov, and L. I. Korovin, "Theory of reflection and absorption of light by low-dimensional semiconductor objects in a strong magnetic field under monochromatic and pulsed excitations," Physics of the Solid State, vol. 46, no. 9, pp. 1761-1775, 2004, translated from Fizika Tverdogo Tela, vol. 46, p. 1706, 2004.

[13] S. T. Pavlov, I. G. Lang, and L. I. Korovin, in Proceedings of the 15th International Symposium "Nanostructures: Science and Technology", p. 207, 2007.

[14] I. G. Lang, L. I. Korovin, and S. T. Pavlov, "A Semiclassical Method in the Theory of Light Scattering by Semiconductor Quantum Dots," Zhurnal Eksperimental'noi $i$ Teoreticheskoi 
Fiziki, vol. 133, no. 6, pp. 1169-1182, 2008, translated in Journal of Experimental and Theoretical Physics, vol. 106, no. 6, pp. 1019-1032, 2008.

[15] D. A. Contreras-Solorio, S. T. Pavlov, L. I. Korovin, and I. G. Lang, "Magneto-optical effects in quantum wells irradiated with light pulses," Physical Review B, vol. 62, no. 24, pp. 16815$16819,2000$.

[16] L. I. Korovin, I. G. Lang, D. A. Contreras-Solorio, and S. T. Pavlov, "The role of spatial dispersion of an electromagnetic wave in its penetration through a quantum well," Physics of the Solid State, vol. 43, no. 11, pp. 2182-2191, 2001, translated from Fizika Tverdogo Tela, vol. 43, p. 2091, 2001.

[17] I. G. Lang, L. I. Korovin, J. A. de la Ruz-Alkas, and S. T. Pavlov, "Quantum theory of electric conduction of spacially nonuniform systems," Zhurnal Eksperimental'noj i Teoreticheskoj Fiziki, vol. 123, no. 2, pp. 305-325, 2003.

[18] I. G. Lang, L. I. Korovin, J. A. de La Cruz-Alcaz, and S. T. Pavlov, "Quantum theory of conductivity of spatially inhomogeneous systems," Journal of Experimental and Theoretical Physics, vol. 96, no. 2, pp. 268-285, 2003.

[19] I. E. Tamm, The Foundation of Electricity, Nauka, Moscow, Russia, 1966.

[20] S. T. Pavlov, I. G. Lang, and L. I. Korovin, "An analog of the Kubo formula for conductivity of spatially inhomogeneous media in spatially inhomogeneous electric fields," Physics of the Solid State, vol. 45, no. 10, pp. 2001-2011, 2003, translated from Fizika Tverdogo Tela, vol. 45, p. 1903, 2003.

[21] I. M. Tsidilkovsky, The Band Structure of Semiconductors, Nauka, Moscow, Russia, 1978.

[22] S. V. Goupalov and E. L. Ivchenko, "Electron-hole longrange exchange interaction in semiconductor quantum dots," Journal of Crystal Growth, vol. 184-185, pp. 393-397, 1998.

[23] S. V. Gupalov and E. L. Ivchenko, "The fine structure of excitonic levels in CdSe nanocrystals," Physics of the Solid State, vol. 42, no. 11, pp. 2030-2038, 2000, translated from Fizika Tverdogo Tela, vol. 42, p. 1976, 2000.

[24] I. G. Lang, L. I. Korovin, D. A. Contreras-Solorio, and S. T. Pavlov, "A manifestation of the magnetopolaron effect in reflection and absorption of light by a three-level system in a quantum well," Physics of the Solid State, vol. 44, no. 11, pp. 2181-2195, 2002, translated from Fizika Tverdogo Tela, vol. 44, p. 2084, 2002.

[25] L. I. Korovin, I. G. Lang, D. A. Contreras-Solorio, and S. T. Pavlov, "Change in the shape of a symmetric light pulse passing through a quantum well," Physics of the Solid State, vol. 42, no. 12, pp. 2300-2313, 2000, translated from Fizika Tverdogo Tela, vol. 42, p. 2230, 2000.

[26] L. I. Korovin, I. G. Lang, D. A. Contreras-Solorio, and S. T. Pavlov, "Penetration of a symmetric light pulse through a wide quantum well," Physics of the Solid State, vol. 44, no. 9, pp. 1759-1768, 2002, translated from Fizika Tverdogo Tela, vol. 44, p. $1681,2002$.

[27] L. I. Korovin, I. G. Lang, and S. T. Pavlov, "Resonant transmission of a light pulse through a quantum well," Physics of the Solid State, vol. 50, p. 341, 2008, translated from Fizika Tverdogo Tela, vol. 50, p. 328, 2005. 

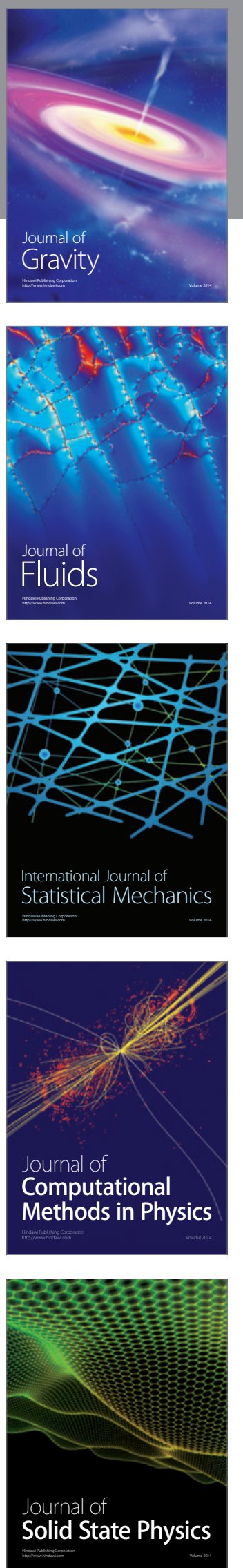

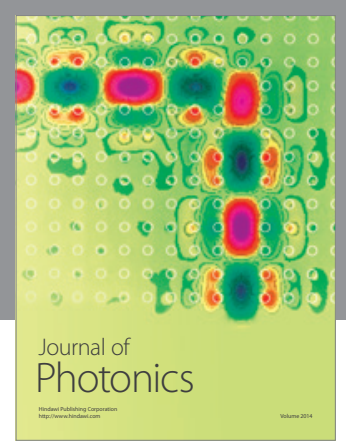

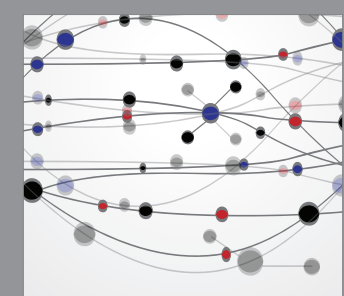

The Scientific World Journal
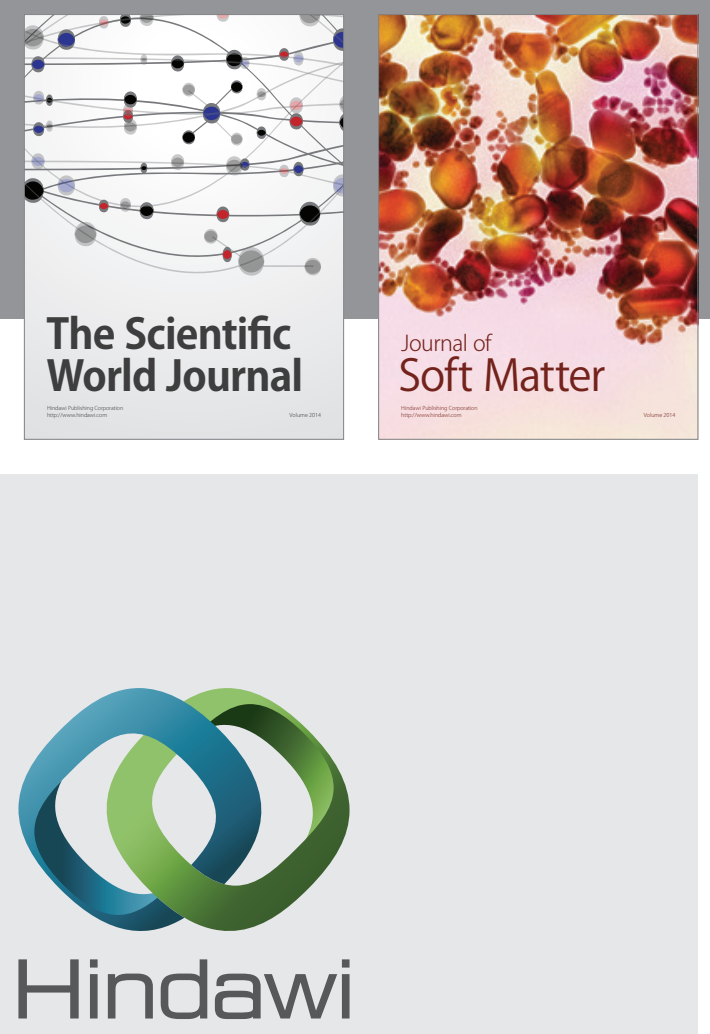

Submit your manuscripts at

http://www.hindawi.com
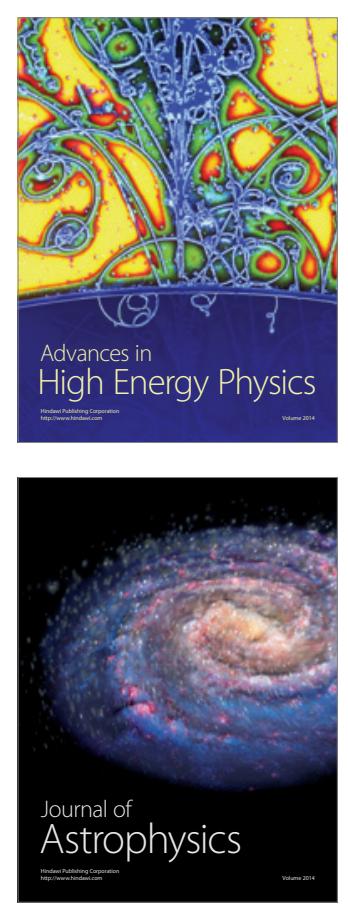
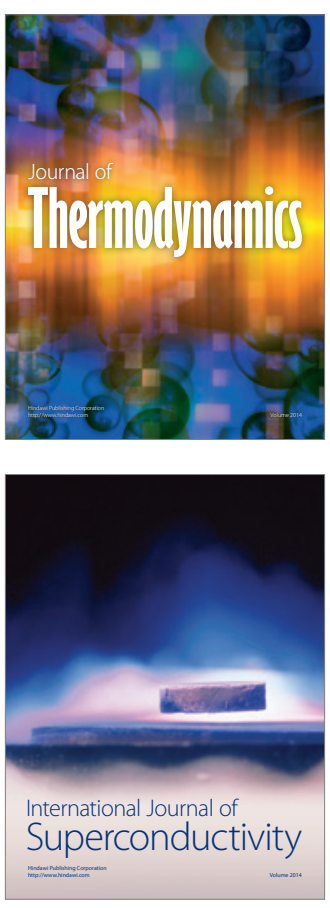
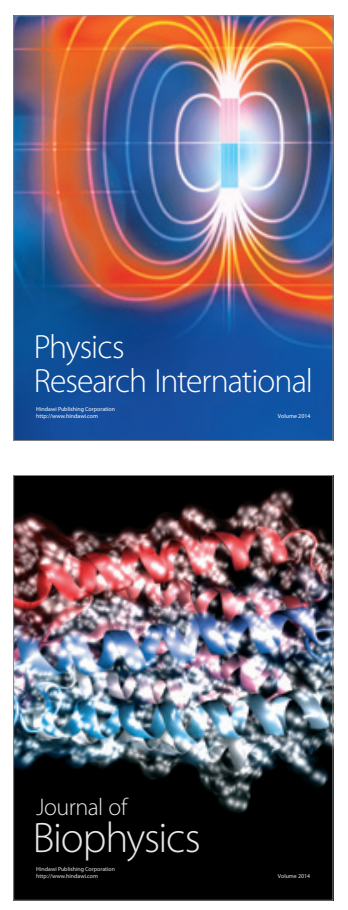
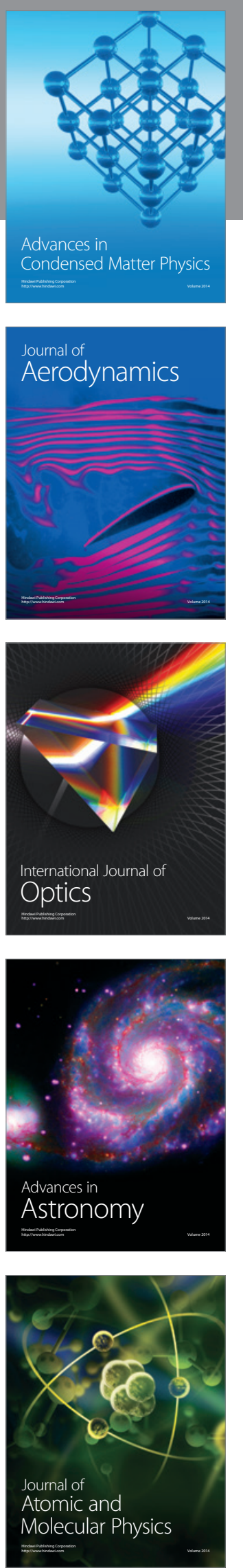